\title{
Les étagères Lipman
}

Fleuron d'une bibliothèque moderne à la veille de la Première Guerre mondiale

\section{Aude Therstappen}

\section{(2) OpenEdition}

\section{Journals}

Édition électronique

URL : https://journals.openedition.org/rbnu/3490

DOI : $10.4000 /$ rbnu.3490

ISSN : 2679-6104

Éditeur

Bibliothèque nationale et universitaire de Strasbourg

\section{Édition imprimée}

Date de publication : 1 mai 2011

Pagination : 80-83

ISSN : 2109-2761

\section{Référence électronique}

Aude Therstappen, "Les étagères Lipman », La Revue de la BNU [En ligne], 3 | 2011, mis en ligne le 01 mai 2011, consulté le 09 août 2021. URL : http://journals.openedition.org/rbnu/3490 ; DOI : https:// doi.org/10.4000/rbnu.3490

\section{(9) $(0 \Theta \odot$}

La Revue de la BNU est mise à disposition selon les termes de la Licence Creative Commons Attribution - Pas d'Utilisation Commerciale - Partage dans les Mêmes Conditions 4.0 International. 


\section{LES ÉTAGÈRES LIPMAN,}

\section{fleuron d'une bibliothèque moderne à la veille de la Première Guerre mondiale}

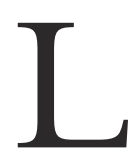

es étagères Lipman, qui équipaient la Kaiserliche Universitäts- und Landesbibliothek (ancêtre de la BNU) à l'ouverture de son nouveau bâtiment en 1895 (et qui seront remontées dans deux étages de magasins dans le bâtiment rénové en 2014) portent le nom de leur inventeur, Robert Lipman. Serrurier de profession, celui-ci développa un système astucieux : il imagina une étagère en métal dont les planches en bois avaient toutes une longueur d'un mètre. On pouvait fixer ces planches aux étagères et régler facilement leur hauteur, grâce à un système de crans dans lesquels les fixations des planches venaient s'emboîter. On pouvait aussi, en fonction des nouveaux arrivages, déplacer la planche en laissant les livres en place, dans leur classement original, ce qui évitait un grand travail de rangement aux magasiniers. Des ardoises placées sur les côtés des étagères permettaient d'inscrire à la craie les cotes des livres rangés et de les actualiser au fur et à mesure des ajustements.

C'est à Strasbourg que ce système a été utilisé pour la première fois. Un nouveau procédé a même été mis en place pour la bibliothèque : les piliers des étagères en métal traversaient les étages, devenant ainsi des éléments statiques à part entière, participant à l'équilibre de l'ensemble du bâtiment. Le poids des livres était ainsi réparti sur tous les étages. L'inconvénient majeur de cette solution est que les étagères ne peuvent ultérieurement plus jamais être bougées. Le magasin est en quelque sorte figé. Très répandues jusque dans les années cinquante, les étagères Lipman furent ensuite peu à peu remplacées par le système des compactus, montés sur des rails et collés les uns aux autres, ce qui permet un gain de place optimal. 


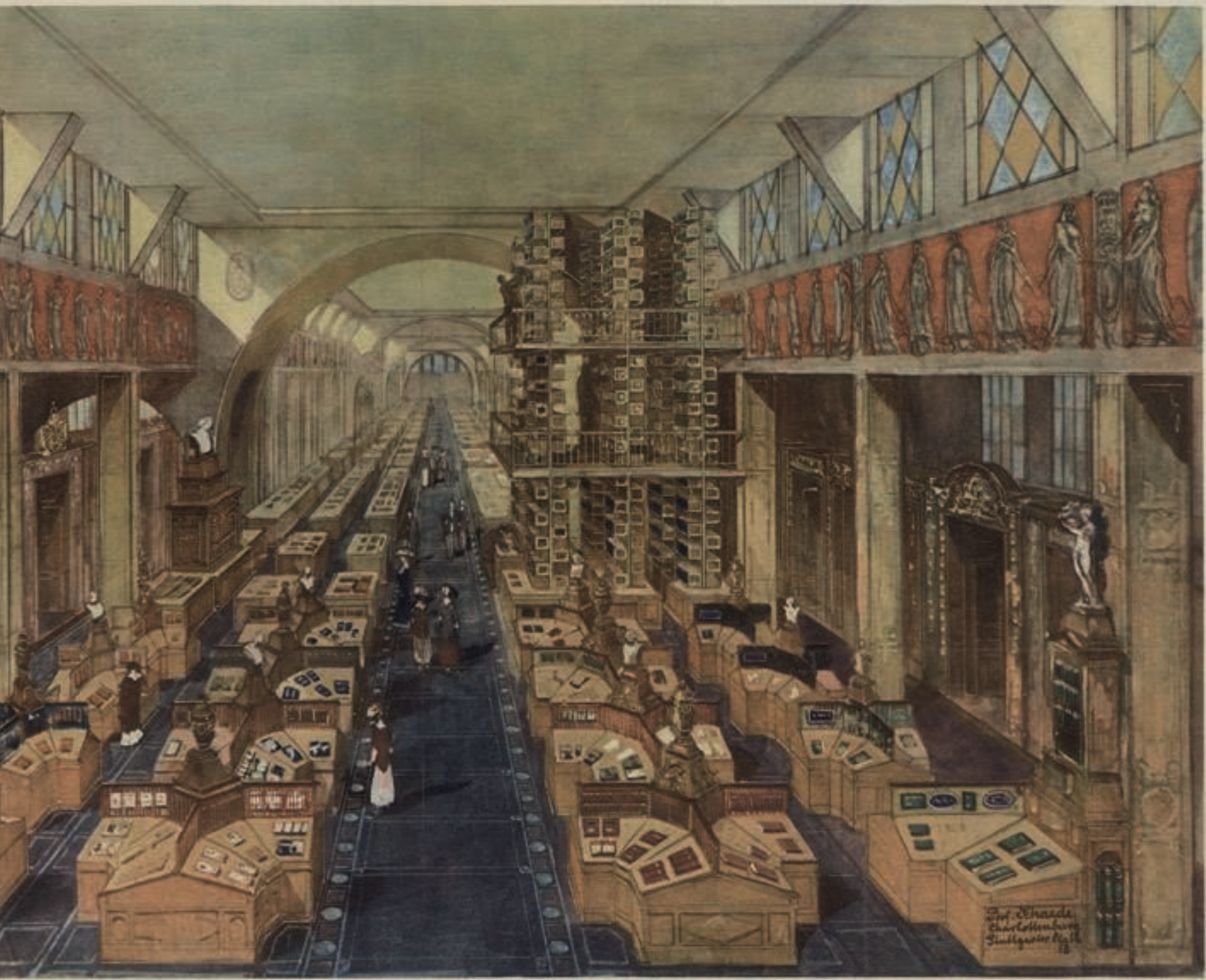

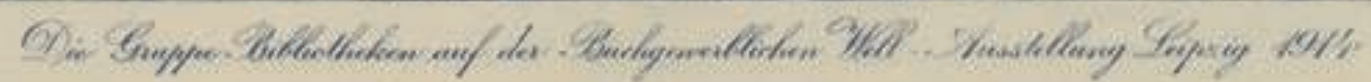

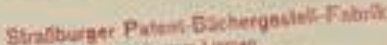
Wear Netter a jacobit? bence we.

Vue de la section consacrée aux bibliothèques à la BUGRA de Leipzig en 1914 (archives BNU). On reconnaît, au fond à droite, le système Lipman. 
Fleuron de l'industrie alsacienne, les étagères Lipman étaient commercialisées par l'entreprise Wolf Netter \& Jacobi. Réputée dans son domaine, dotée de deux sièges à Strasbourg et à Berlin, elle fut chargée d'aménager le pavillon des bibliothèques de la grande exposition internationale des métiers du livre et des métiers graphiques (Ausstellung für Buchgewerbe und Graphik, aussi nommée BUGRA) de Leipzig en 1914. Ce choix témoigne de la place importante occupée par cette entreprise et ses produits, considérés comme à la pointe des techniques modernes d'aménagement des bibliothèques. Le projet de l'exposition était très ambitieux : il ne s'agissait de rien moins que de présenter l'ensemble des arts et métiers en lien avec le livre et le graphisme, aussi bien dans leur développement historique que dans leurs développements techniques et intellectuels actuels. Le projet du pavillon des bibliothèques n'était pas moins universel puisqu'il avait pour but de réaliser un panorama complet du monde des bibliothèques, à la fois pour le grand public et pour le public spécialisé. Le directeur de la Kaiserliche Universitätsund Landesbibliothek, le Dr. Wolfram, faisait partie du comité d'organisation de la section consacrée aux bibliothèques.

Tous les aspects du travail devaient être abordés et la liste initiale conçue par le comité est impressionnante par la diversité et le degré de détail des éléments présentés. Il s'agissait en effet de permettre au visiteur d'appréhender un univers à la fois en gros (plans, vues et photographies des bibliothèques, reconstitution d'une bibliothèque miniature) mais aussi de le comprendre dans son fonctionnement interne (aménagement des salles de lecture et des banques de prêt, meubles de stockage pour les journaux et les cartes, ascenseurs, chariots à livres, matériel de dépoussiérage, aspirateurs). Tout ce qui était alors utile au travail en bibliothèque était exposé : étiquettes et présentation des livres en libre accès, cartes de lecteurs, réservations, prêt entre bibliothèques, retards... Le cœur du travail des bibliothécaires était aussi présenté (bibliographie, acquisition des livres, relations avec les libraires, achats, dons, échanges et dépôt légal, journaux et collections automatiques, réclamations et suggestions, catalogues sous forme papier, sur fiches ou en volumes). On décida même de montrer au public différents échantillons de catalogues : alphabétiques,

systématiques, spécialisés, centralisés et conçus par lieu de conservation. Des éléments statistiques sur le personnel, les acquisitions, l'administration ainsi que des règlements de bibliothèques furent également fournis. Certaines vitrines présentaient les productions des ateliers de restauration : travaux de conservation et de restauration, confection de reliures... Même les événements extraordinaires ne sont pas laissés de côté puisqu'il est prévu d'informer le public sur l'organisation des déménagements de bibliothèques (prévision et transport). Un autre secteur concernait la formation et les concours de bibliothèques et présentait les principaux manuels existant alors sur la profession de bibliothécaire. Une section était dédiée aux bibliophiles et collectionneurs avec une sélection de tirages rares, de reliures, de collections d'affiches, d'ex-libris et de timbres. Enfin, une partie retraçait l'histoire des bibliothèques depuis l'Antiquité et présentait des biographies de grands bibliothécaires. La section "bibliothèques " de l'exposition avait si bien atteint le but qu'elle s'était fixé (illustrer le travail des bibliothèques de façon " intéressante et instructive ") que dans une lettre du 23 octobre 1914, la très puissante association des libraires de Leipzig déplorait que les collections soient sur le point d'être dispersées et qu'on ne puisse en profiter pour constituer un musée permanent à la Deutsche Bücherei de Leipzig. La Première Guerre mondiale qui venait d'éclater ne devait pas permettre de concrétiser ce projet et il fut déjà bien difficile de parvenir à retourner les œuvres prêtées à leurs établissements d'origine.

L'illustration de la page 81 nous présente la grande salle consacrée aux bibliothèques, avec ses différentes vitrines thématiques, mais le plus frappant est sans doute la reconstitution d'un magasin de livres constitué, sur trois niveaux, de travées d'étagères Lipman. Réalisation de la technique moderne fièrement présentée dans une exposition où le monde des bibliothèques se célèbre lui-même, ces étagères constituent un objet emblématique, qui allait dominer encore longtemps le paysage des bibliothèques, figé lui aussi dans son développement, comme tant d'autres secteurs, par la Première Guerre mondiale.

\section{Aude Therstappen}



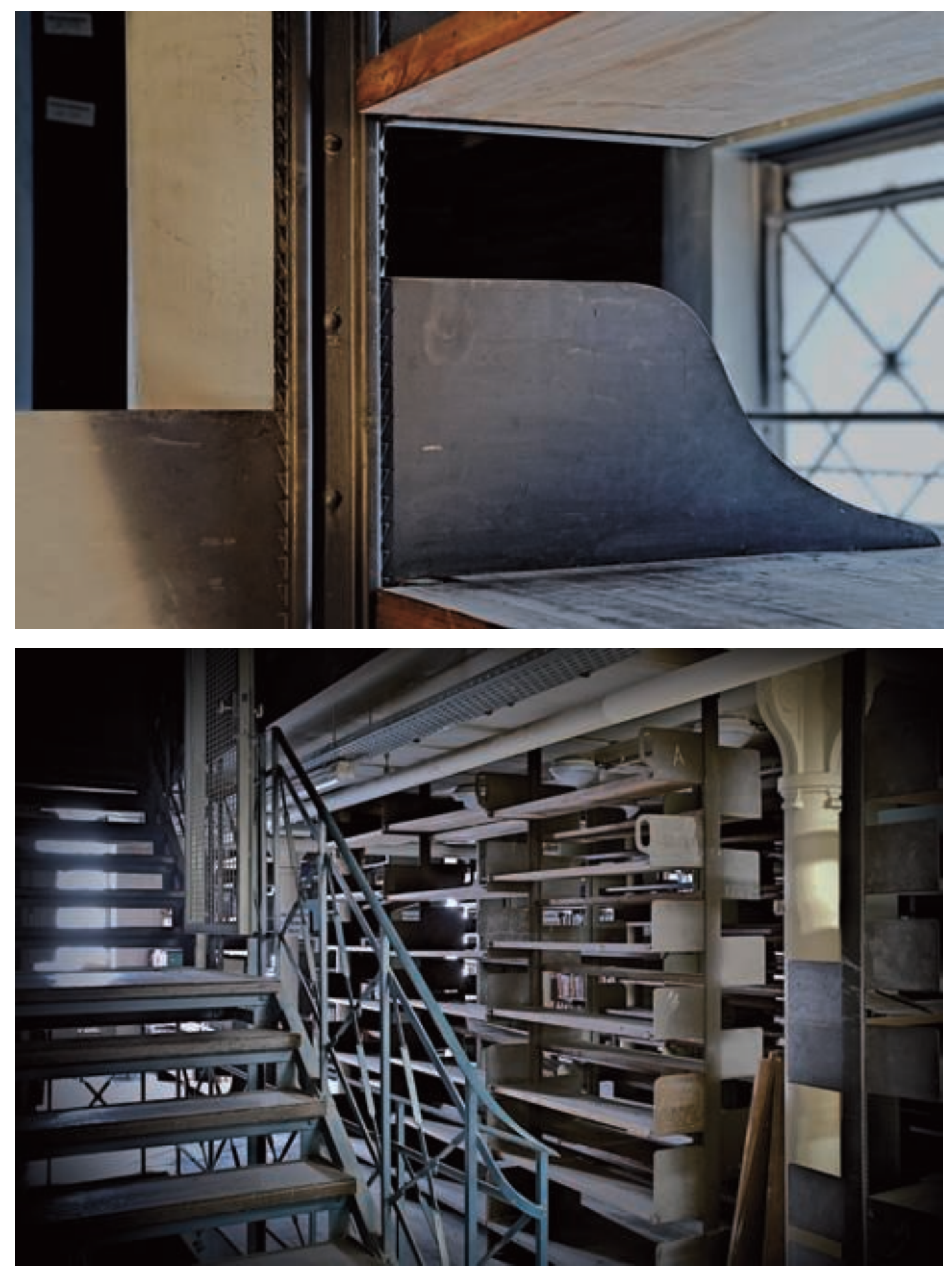

Détail d'une étagère et du système de fixation

Les étagères in situ (bâtiment de la place de la République avant restructuration) 